\title{
Pupil dilation signals recognition salience
}

\author{
Ian G. Dobbins ${ }^{1}$ (1)
}

Accepted: 16 December 2020 / Published online: 15 January 2021

(C) The Psychonomic Society, Inc. 2021

\begin{abstract}
Stimuli that are recognized from a prior encounter elicit larger pupil dilations than those that are not. This study tests an account of this recognition dilation response (RDR) that assumes newly encountered recognition signals, like new percepts, elicit attentional orienting responses. Because orienting is moderated by motivational significance and expectation, the RDR was tested for these properties; manipulating incentives for "old" versus "new" judgments, and isolating the effects of runs of "old" versus "new" decisions on the subsequent RDR, in two experiments. Whereas incentivizing "new" decisions largely eliminated the RDR, incentivizing "old" decisions amplified it. Moreover, the RDR was prominent following runs of "new" decisions, yet minimal following runs of "old" decisions. Thus, the pupil dilates more as recognition memory becomes more valuable and/or unexpected. This recognition-orienting response was functionally separate from an additional, late dilation linked to feedback expectancy. Thus, the pupil separately signals the salience of recognition evidence, and the expectation of post-decision feedback.
\end{abstract}

\section{Introduction}

In addition to reflexive increases following reduced light levels, the pupil also dilates as cognitive "effort" increases (Kahneman \& Beatty, 1966). For example, pupil dilation increases with increases in short-term memory set size (Kahneman, 1973; Kahneman \& Beatty, 1966), and the complexity of mental arithmetic (Hess \& Polt 1964; for review, see Beatty, 1982). Under the cognitive-load framework, the primary basis of these dilations is the time-pressure under which cognitive operations, such as rehearsal and calculation, occur, with Kahneman (1973) noting:

“...severe time-pressure necessarily arises in any task which imposes a significant load on short-term memory, because the subject's rate of activity must be paced by the rate of decay of the stored elements. In mental arithmetic, for instance, one must keep track of the initial problem, of partial results already obtained, and of the next step. Stopping or slowing even for an instant usually forces one to return to the beginning and start again" (p. 26).

Under this formulation cognitive load cannot be simply reduced to the amount of information processed since even large amounts will involve minimal time-pressure if leisurely processing is possible. Kahneman (1973) referred to such processing as

Ian G. Dobbins

idobbins@wustl.edu

1 Department of Psychological and Brain Sciences, Washington University in Saint Louis, Saint Louis, MO 63130, USA "mental walking" to contrast it with hurried mental "jogging" of time-pressured cognition. Load also cannot be reduced to task difficulty, defined as probability of success, because exceedingly difficult tasks can nonetheless require minimal cogitation. For example, successfully guessing a number between 1 and 1,000 is difficult but requires minimal information processing. Kahneman (1973) referred to time-pressured deliberative cognition, which places a demand or load on a fixed capacity of attentional resources, as "voluntary effort."

Given the established link between pupil dilation and cognitive effort, it is surprising, however, that episodic recognition also dilates the pupil, yielding greater dilation for stimuli that are recognized versus correctly rejected (Heaver \& Hutton, 2011; Mill et al., 2016; Otero et al., 2011; Papesh et al., 2012; Võ et al., 2008). The functional interpretation of this recognition dilation response (RDR) ${ }^{1}$ remains a mystery because successful recognition does not seem to require greater time-pressured deliberation compared to the rejection of new items. Indeed, hits are not only typically more certain than correct rejections (Dobbins \& Han, 2007), but are typically rendered more quickly, as will be shown in the current report. Moreover, deepening levels of processing at encoding increases the later RDR (Otero et al., 2011). Since deep materials are more accurately, rapidly, and confidently identified than shallow materials, it would be odd to claim they require more deliberative "effort" than correct rejections. Based on this line

\footnotetext{
${ }^{1}$ Võ et al. (2008) referred to this effect as the pupil old/new effect. Here the term recognition dilation response (RDR) is used because the pupil dilates both when recognition is accurate (viz., hits) and mistaken (viz., false alarms) (Mill et al., 2016; Otero et al., 2011), even though in the latter case, the dilation is in response to new materials.
} 
of reasoning, Otero and colleagues rejected a cognitive load interpretation of the RDR, instead suggesting it might reflect the overall strength of recognition evidence.

However, the cognitive-load framework also distinguished the "voluntary effort" of deliberative cognition from the "involuntary effort" of the orienting response (Kahneman 1973). The orienting response is a collection of physiological reactions, most notably pupillary dilation, that occur rapidly and involuntarily following environmental changes (Sokolov, 1963; Sokolov et al., 2002). As with time-pressured deliberative cognition, orienting responses are also assumed to draw upon on a limited capacity of attentional resources and are hence effortful in this general sense. Because deliberative cognition and orienting responses reflect different psychological phenomena, Kahneman warned against conflating them when interpreting the functional significance pupil dilations (p. 48). To limit confusion here, I will reserve the term "cognitive effort" for deliberative, timepressured explicit cognition, and will refer to orienting responses as such, avoiding the term "involuntary effort" since it is somewhat oxymoronic. This also avoids implying that orienting responses are effortful in the generally understood sense. ${ }^{2}$

Critically, orienting responses are most pronounced for unexpected stimuli (Friedman, Hakerem, Sutton, \& Fleiss, 1973; Nieuwenhuis, Geus, \& Aston-Jones, 2011), and they are enhanced for stimuli that are increasingly motivationally significant (for review, see Bradley, 2009). The current study tests the RDR for these properties based on the anecdotal evidence that most individuals have had their attention involuntarily captured by stumbling upon a familiar face in some unexpected context, for example while traveling (Baddeley, 1997; Mandler, 1980). Initial experimental support for this memory-triggered orienting hypothesis was also provided by Mill, O'Connor, and Dobbins (2016), who used explicit, predictive cues to show that the RDR was enhanced when recognition was unexpected, and essentially eliminated when recognition was expected. Thus, the size of the RDR (hit dilation > correct rejection dilation) depended upon subjective expectations, which is a hallmark of orienting responses.

However, the findings of Mill, O'Connor, and Dobbins (2016) raised two questions. First, if the RDR is an orienting response and thus moderated by expectations, why is it present in standard recognition memory tasks that do not explicitly manipulate expectations (e.g., Heaver \& Hutton, 2011; Mill et al., 2016; Otero et al., 2011; Võ et al., 2008)? Second, does the RDR show the other hallmark of the orienting response, namely, its sensitivity to the motivational significance of eliciting stimuli (Failing \& Theeuwes, 2014; Le Pelley et al., 2016; Matsumoto \& Hikosaka, 2009; Nieuwenhuis et al., 2011)?

\footnotetext{
${ }^{2}$ For example, Merriam-Webster.com defines effort as the "conscious exertion of power" or "a serious attempt," whereas Google.com defines it as "a vigorous or determined attempt" or "the result of an attempt."
}

This study addresses these questions using a novel manipulation and analysis during recognition memory accompanied by pupillometry. First, the motivational significance of recognition evidence was manipulated by altering whether "old" or "new" conclusions offered the possibility of winning (or losing) points applied towards a final candy payout and fictive memory competition. It was predicted that incentivizing "old" decisions would potentiate the ability of recognition evidence to trigger attentional orienting, whereas incentivizing "new" decisions (which reflect the lack of recognition evidence) would minimize this ability. Second, in a control test of standard recognition without incentives, each trial was coded as a function of the two immediately preceding recognition decisions. This novel coding scheme allowed the comparison of the RDR following runs of "new" versus "old" conclusions and was motivated by the fact that orienting responses are increased for violations of local expectations (for detail, see Squires et al., 1976). The prediction was that the RDR would be potentiated following runs of "new" versus "old" conclusions, because during the former, local expectations of recognition are low. There were two experiments. In Experiment 1 feedback was provided intermittently during the incentivized tests, whereas Experiment 2 eliminated all response feedback.

\section{Methods}

\section{Participants}

In Experiment 1, 39 undergraduate students (nine male; mean age 19.5 years; range 18-22) participated and in Experiment 2, 44 undergraduate students participated (13 male; mean age 19.6 years; range 18-22). Subjects participated in exchange for course credit in accordance with Washington University in St. Louis Internal Review Board guidelines. All subjects had normal or corrected-to-normal vision and chosen sample sizes were consistent with the Mill, O'Connor, and Dobbins (2016) pupillometry study, yielding adequate power based on that observed effect. ${ }^{3}$ Following pupillometry trial rejection procedures (see below), 36 of the 39 subjects were retained from

\footnotetext{
${ }^{3}$ Power was estimated via Monte Carlo simulation based on the dilation effect observed in Mill, O'Connor, and Dobbins (2016). This study examined the effect of anticipatory cues on the RDR and yielded a reliable interaction between Cue (Likely Old vs. Likely New) and Outcome (hit vs. correct rejection) on mean percent dilation. Assuming the current incentive manipulation is as effective as anticipatory cueing at moderating the RDR, I used the condition means and covariance matrix from those data as parameters for a multivariate normal effects distribution which was then randomly sampled from 1,000 times at the current sample sizes. Following each sample, the data were analyzed with a Cue by Outcome $2 \times 2$ ANOVA and the main effects and interaction p-values were recorded. For the final sample size of 36 in the current Experiment 1, the simulation indicated a power of .86 for detecting the interaction. For the Experiment 2 sample size of 43 the power was .98. Thus, if incentives have comparable effects to cued expectations on the RDR, both experiments of the current study were sufficiently powered to detect the interaction.
} 
Experiment 1, and 43 of the 44 subjects were retained from Experiment 2.

\section{Equipment and materials}

Pupillometry was recorded using an EyeLink 1000 (SR Research, Mississauga, Canada) sampling at $1,000 \mathrm{~Hz}$ controlled by PsychoPy (Peirce, 2007) and PyGaze (Dalmaijer, Mathôt, \& Van der Stigchel, 2014). Recordings were from the left eye with subjects seated approximately $55 \mathrm{~cm}$ from the screen using a chin rest. The eye tracker was calibrated using a nine-point procedure prior to the first recognition phase. The maximum error allowance for a single point was $1.0^{\circ}$ and the average gaze error across the nine fixation points was $0.5^{\circ}$ or less.

Stimuli consisted of 300 common nouns with an average of 6.76 letters, 2.37 syllables, and 34.5 occurrences per million printed word frequency (Kucera, Kučera, \& Francis, 1967). From this set, three random lists of 100 items (50 studied, 50 novel) were created for each participant for three recognition tests. Stimuli were presented in white using Ariel font at an approximately 51-point size, against a gray background with all other room illumination off.

\section{Procedure}

There were two study phases and three test phases (see Fig. 1). Study Phase 1 had 50 items and was immediately followed by a standard 100-item recognition test without feedback (Test 1). This established the baseline RDR in the absence of any mention of incentives or provision of feedback, and also served as the data for the analysis of sequential decisions effects on the RDR under basic recognition-testing conditions. Thus, the first test in both experiments serves as a manipulation check for the basic RDR and provides data for examining potential sequential decision effects on the RDR. Study Phase 2 contained 100 items and supported two subsequent 100-item recognition tests, counterbalanced for order across the two incentive conditions (see Fig. 1). Importantly, there was no mention of incentives until after Study Phase 2. Thus, all the targets encoded for the "old" and "new" incentives tests were encoded identically, within a single study list, and without the subject's foreknowledge of the upcoming incentive manipulations. Given this design, any pupillometry differences observed across the incentivized tests would necessarily reflect the operation of the incentive manipulation occurring at test.

During encoding, participants performed a self-paced syllable-counting task on serially presented words that they were instructed to remember for an upcoming memory test. Participants pressed the "Z" key for words with one or two syllables and the "M" key for words with three or more syllables. This orienting task yields moderate levels of subsequent recognition discrimination.
During each recognition test, 100 words (50 studied, 50 unstudied) were randomly mixed and presented serially. Each trial began with a centered fixation pattern (XXXXX) for 2,000 ms, followed by a test probe for 4,000 ms during which participants decided whether the word was "old" or "new" by pressing the "Z" or "M" key, respectively. Confidence was registered by the number of key presses, with one, two, or three presses used to indicate low, medium, or high confidence, respectively.

Following Study Phase 2, the incentive manipulation was explained. For the new-incentivized test, participants were informed that they would gain 20 points for correct "new" responses (i.e., correct rejections) and lose 20 points for incorrect "new" responses (i.e., misses). It was emphasized that they would not gain or lose points for "old" responses. For the old-incentivized test, the incentives were applied to correct and incorrect "old" responses (i.e., hits and false alarms), and the instructions emphasized that "new" responses would not gain or lose points. Both incentive conditions reflect a neutral payout scheme and were not expected to alter decision bias or accuracy (Macmillan \& Creelman, 2004). For example, when "old" responses are incentivized, relaxing the old/new decision criterion would not only increase hits (increasing point totals) but also false alarms (decreasing point totals). Given this, ideally subjects should maintain a neutral decision bias regardless of whether responses are incentivized or not. ${ }^{4}$ Participants were informed that their points would be translated into a candy payout at the end of the study and a bowl of candy remained in plain sight. To further incentivize participants, a "hall of fame" manipulation was used. Akin to an arcade game, a list of high scores was presented and participants were prompted to enter a username that would be displayed in the hall of fame if their point total warranted it. Neither their username nor score was recorded and all subjects received the same candy payout at the end of the experiment. A unique, fictive list of high scores preceded each of the two incentivized tests.

In Experiment 1, during the incentivized tests, a random $15 \%$ of incentive consistent responses (i.e., "old" in old incentivized test, and "new" in new incentivized test) was followed by $1,000 \mathrm{~ms}$ of feedback, indicating response accuracy and the current point total. On non-feedback trials a blank screen was displayed for 1,000 ms. Participants were told that most responses would not receive feedback but that points would nonetheless accrue. In Experiment 2, feedback was eliminated during the incentivized tests and participants only saw their point total at the end of each incentivized test.

\footnotetext{
${ }^{4}$ In behavioral economics, the current incentive manipulation might instead be characterized as a manipulation of risk because it increases the variance in reward versus punishment for the two outcomes (success or failure). Regardless, it would nonetheless serve to increase the motivational significance of recognition.
} 


\begin{tabular}{ccc}
\hline Procedure & Incentive Manipulation & Method \\
\hline STUDY Phase 1 & & 50 words (syllable counting) \\
Recognition TEST 1 & $\begin{array}{c}\text { No reward or feedback } \\
\text { manipulation explained }\end{array}$ & 100 words (50 old/50 new) \\
STUDY Phase 2 & & 100 words (syllable counting) \\
\hline Instruction & Potential for reward explained & Points, candy \& high-score hall of fame \\
\hline Recognition TEST 2 & 'New' responses incentivized & 100 words (50 old/50 new) \\
Recognition TEST 3 & 'Old' responses incentivized & 100 words (50 old $/ 50$ new) \\
\hline
\end{tabular}

Fig. 1 Design of the experiments. The order of the incentive condition in Tests 2 and 3 was counterbalanced across participants

\section{Pupillometry preprocessing and statistics}

Blinks and artifacts were identified through signal dropout in each trial's dilation time course indicated by NA values. Trials missing in excess of $15 \%$ of the baseline or probe period were discarded, along with trials missing a response. Subjects losing more than $33 \%$ of trials were discarded, leaving 36 subjects in Experiment 1, and 43 in Experiment 2. For these remaining subjects, there was an average loss of $7 \%$ of trials in Experiments 1 and 2.

For retained trials, following linear interpolation, the data were down-sampled to $20 \mathrm{~Hz}$ by averaging every 50 samples. Finally, each trial was baseline corrected by subtracting the mean dilation during the 1,000 -ms baseline period from the trial dilation time course. Then, these dilation time courses were normalized by dividing them by the grand mean of all the baseline periods for each participant (Mathôt et al., 2018).

For statistical inference, the mean dilation during hits and correct rejections from probe onset to $4,000 \mathrm{~ms}$ were analyzed with omnibus ANOVAs. Following this, the temporal specificity of the effects was determined by pairwise comparison of dilations during hits versus correct rejections at each 50-ms time point within each condition of interest. These pairwise tests were corrected for multiple comparisons using the False Discovery Rate (FDR) procedure (Benjamini \& Hochberg, 1995) thresholded at .05 for the 81 timepoints. Data were analyzed in the R language ( $\mathrm{R}$ Core Team, 2013) using the tidyverse (Wickham, 2017), broom (Robinson, 2017), ez (Lawrence, 2016), and forcats (Wickham, 2019) packages.

\section{Results}

\section{Behavior}

Subject accuracy and decision bias were calculated using d' and $\mathrm{C}$ signal detection measures except where noted (Macmillan \& Creelman, 2004). Because the control test always took place first and used a study list half as long as that preceding the latter two incentivized tests, it will be considered separately from the incentivized tests (see Methods for justification). Analysis of confidence and reaction times are in the Online Supplementary Material.

Experiment 1 - behavior Mean accuracy and bias in the initial control test were $1.50(\mathrm{SD}=.58)$ and $.03(\mathrm{SD}=$ $.10)$, respectively. Turning to the latter incentivized tests, accuracy was similar when "old" $(\mathrm{M}=1.29, \mathrm{SD}=.48)$ versus "new" $(\mathrm{M}=1.15, \mathrm{SD}=.52)$ decisions were incentivized $(\mathrm{t}(35)=1.72, \mathrm{p}>.09)$, and bias was also similar when "old" $(\mathrm{M}=.14, \mathrm{SD}=.12)$ versus "new" $(\mathrm{M}=.03, \mathrm{SD}=.07)$ decisions were incentivized $(\mathrm{t}(35)=1.53, \mathrm{p}>.13)$.

Experiment 2 - behavior During Experiment 2, accuracy and bias in the control test were $1.16(\mathrm{SD}=.46)$ and $.04(\mathrm{SD}=$ .07), respectively. Turning to the latter incentivized tests, accuracy was similar when "old" $(\mathrm{M}=1.21, \mathrm{SD}=.41)$ versus "new" $(\mathrm{M}=1.16, \mathrm{SD}=.46)$ decisions were incentivized $(\mathrm{t}(42)=.72, \mathrm{p}>.47)$. Bias was also similar when "old" $(\mathrm{M}=.00, \mathrm{SD}=.12)$ versus "new" $(\mathrm{M}=.04, \mathrm{SD}=.07)$ decisions were incentivized $(\mathrm{t}(42)=.69, \mathrm{p}>.49)$.

The behavioral data of Experiment 2 largely replicated Experiment 1. Neither discrimination accuracy nor bias were reliably affected by the incentive manipulation, and hits were generally more certain and more rapid than correct rejections (see Online Supplementary Material).

\section{Pupillometry}

Figure 2 shows the dilation time courses for hits and correct rejections in each of the tests (control/no incentives, "new" incentivized, and "old" incentivized) for Experiments 1 and 2 (Fig. 2a, b). The analyses begin with Experiment 1. As with the behavioral data, because the control test always occurred first and its study list contained half the number of items as the incentivized tests, it is considered it in isolation (see Methods for justification).

During the initial control test, mean dilation during hits was greater than mean dilation during correct rejections $(\mathrm{t}(35)=$ $4.13, \mathrm{~d}=.69, \mathrm{p}<.001)$, demonstrating the standard RDR phenomenon is observed under the current procedures. Pairwise comparisons at each time point indicated the differential RDR began at approximately $900 \mathrm{~ms}$ following probe onset, and 
remained reliable throughout much of the following time course.

Turning to the mean dilation response during incentivized tests, a two-way repeated-measures ANOVA was conducted using factors of Outcome (HITs vs. CRs) and Incentive Condition ("Old"-Incentivized versus "New"-Incentivized). There was a main effect of Outcome $\left(F(1,35)=39.18, \eta_{\mathrm{p}}{ }^{2}=\right.$ $.53, \mathrm{p}<.001)$, no main effect of Incentive Condition $(\mathrm{F}<1)$, and a significant Outcome by Incentive Condition interaction $\left(\mathrm{F}(1,35)=43.26, \eta_{\mathrm{p}}^{2}=.55, \mathrm{p}<.001\right)$. The interaction occurred because hits yielded generally greater dilation than correct rejections during the "old" incentive test (Fig. 2a, rightmost panel), yet during the "new" incentive test, the reverse occurred with correct rejections yielding greater dilation than hits, but only late in the trial (Fig. 2a, middle panel). This was confirmed via pairwise comparisons at each time point. During the "old" incentive test hits yielded greater dilation than correct rejections beginning at $550 \mathrm{~ms}$, becoming consistent at $750 \mathrm{~ms}$, and persisting throughout the remainder of the trial. In contrast, during the "new" incentive test there was no time point at which hits yielded greater dilation than correct rejections and the dilation response was statistically equivalent for hits and correct rejections until approximately 2,800 ms. Thereafter, the reverse of the RDR occurred, with greater dilation for correct rejections than hits.
These data are the first to show that the RDR heavily depends upon decision incentives. Comparison of the "new" and "old" incentive tests clearly demonstrates that the RDR was only present in the latter, and it appeared considerably more robust than during the initial control test (cf. Fig. 2a, left vs. right panels). However, the data during the incentivized tests also show a late-occurring dilation beginning at $2,800 \mathrm{~ms}$, which is well after the decision times of either hits $(\mathrm{M}=$ $1,541 \mathrm{~ms}$ and $\mathrm{M}=1,351 \mathrm{~ms})$ or correct rejections $(\mathrm{M}=$ $1,574 \mathrm{~ms}$ and $\mathrm{M}=1,430 \mathrm{~ms}$ ) in the "new" and "old" incentive tests, respectively. This late dilation occurs for correct rejections when "new" responses are incentivized (Fig. 2a, middle panel) and instead occurs for hits when "old" responses are instead incentivized (Fig. 2a, right panel). This late dilation is wholly absent during the initial control test (Fig. 2a, left panel), and therefore must reflect a consequence of either the feedback and/or incentives. This raises the possibility that the late dilation response reflects either the commission of a decision (regardless of whether it is "old" or "new") that is linked to potential reward, or the anticipation of reward feedback regarding that decision. Experiment 2 tested these two possibilities by repeating the experiment with one change: the removal of all decision feedback during the incentive tests.

Figure $2 \mathrm{~b}$ shows the results of Experiment 2. It replicates the incentive effects on the early RDR discovered in Experiment 1.
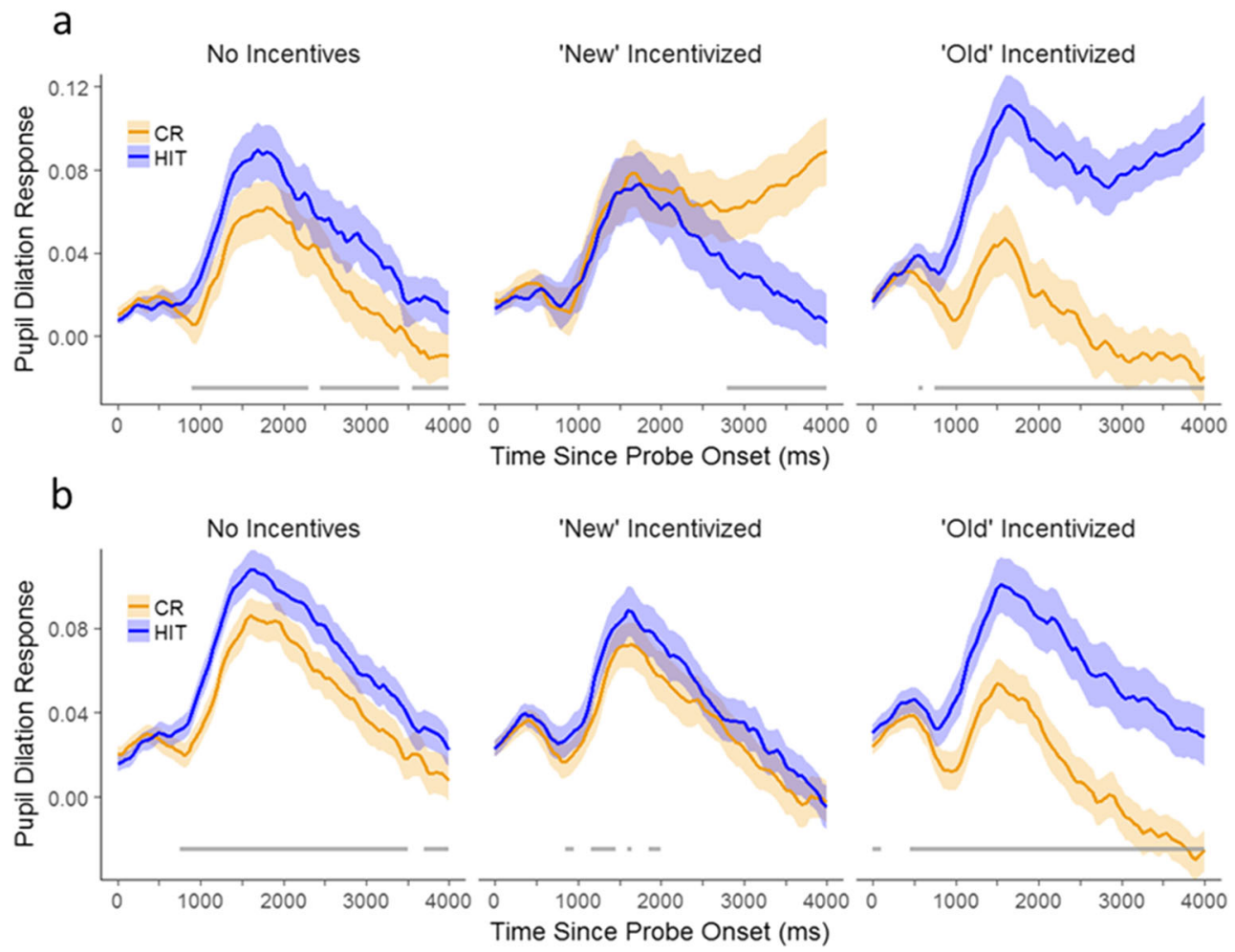

Fig. 2 Recognition dilation time courses for Experiment 1 (a) and Experiment 2 (b). Dilation during hits (HIT) is shown in blue and correct rejections $(\mathrm{CR})$ in orange. Shading illustrates \pm 1 SEM. Left panels illustrate the dilation time courses for the control block in which no incentives were in place. Middle and right panels illustrate the dilation time courses when either "new" or "old" decisions were incentivized, respectively. The gray line along the bottom of the plots indicate timepoints where dilation for hits and correct rejections differ significantly 
During the control test there is greater mean dilation for hits versus correct rejections $(\mathrm{t}(42)=4.51, \mathrm{~d}=.69, \mathrm{p}<.001)$, illustrating the basic RDR phenomenon that begins fairly early at approximately $750 \mathrm{~ms}$. During the later incentivized tests a repeated-measures Outcome by Incentive Condition ANOVA revealed a main effect of Outcome $\left(\mathrm{F}(1,42)=53.01, \eta_{\mathrm{p}}{ }^{2}=.56\right.$, $\mathrm{p}<.001)$, no main effect of Incentive Condition $(\mathrm{F}<1)$, and an Outcome by Incentive Condition interaction $(F(1,42)=18.19$, $\left.\eta_{\mathrm{p}}{ }^{2}=.30, \mathrm{p}<.001\right)$. The latter again demonstrates that the RDR was reliably larger in the "old" versus "new" incentive tests. The comparisons at each time point again demonstrated that the RDR started early in the trial and remained robust during both the control and "old" incentive tests. In the case of the "new" incentive test, the RDR was largely unreliable.

Critically, Experiment 2 also demonstrated the complete absence of the late dilation response demonstrated in Experiment 1 (Fig. 2a vs. Fig. 2b, middle and right panels). Thus, when decision feedback was removed, there was no late, post-response dilation in the incentive tests. This demonstrates that the late dilation response in Experiment 1 reflected the anticipation of feedback for incentivized responses, and not the execution of an incentivized response per se. Moreover, it demonstrates that this feedback anticipation dilation is functionally dissociable from the earlier RDR, which behaves similarly in Experiments 1 and 2.

The next aspect of the memory orienting explanation considered was the sensitivity of the RDR to expectations. If expectations accrue inductively as experience within a given context unfolds, then runs of "old" versus "new" decisions will have different effects on subsequent dilation responses. More specifically, the RDR should be heightened following runs of "new" conclusions, where subsequent recognition is unexpected, versus runs of "old" conclusions where subsequent recognition is expected.

To test this possibility, the data were combined from the initial control tests in Experiments 1 and 2, which are free from incentive-induced effects. ${ }^{5}$ Each trial was coded for the two immediately preceding recognition decisions (regardless of accuracy) yielding four possible sequences of prior decisions (i.e., Old_Old, New_New, Old_New, \& New_Old) where the first label is the decision two trials back, and the second the decision one trial back. Because old and new stimuli are randomly intermixed, each of the four sequence conditions represent approximately $25 \%$ of the trials for an unbiased observer. Two subjects were removed because they did

\footnotetext{
5 This analysis was planned following exploratory plotting of the Experiment 1, Test 1 data, which was conducted prior to Experiment 2. The first statistical tests (ANOVA on means and pairwise time-point comparisons) were conducted on the combined data of Experiments 1 and 2 and were planned. As a reminder, subjects in both experiments were treated identically up to the provision of the instructions for Test 2, at which point the incentive and/or feedback manipulations were introduced. Thus, combining the initial control tests, which were identical, basic recognition tests with the same trial structure and study history is warranted and increases power.
}

not have either hits or correct rejections following one of the sequences. Figure 3 demonstrates that the patterns of prior decisions influenced the current RDR, which was maximal following two "New" conclusions (left) with numerous time points demonstrating a reliable difference. In contrast, the RDR was minimal following two "Old" conclusions (right), without a single time point showing a reliable RDR. The RDR was intermediate in the remaining two sequence conditions. To statistically confirm this impression, the Old_Old and New_New conditions (where the response was anticipated to minimal and maximal) were used in a two-way repeated-measures ANOVA with factors of Outcome (hit vs. correct rejection) and Sequence (Old_Old vs. New_New) conducted on the mean dilation response. The ANOVA yielded a main effect of Outcome $\left(\mathrm{F}(1,76)=12.54, \eta_{\mathrm{p}}{ }^{2}=.14, \mathrm{p}<.001\right)$, no main effect of Sequence $(\mathrm{F}<1)$, and an Outcome by Sequence interaction $\left(\mathrm{F}(1,76)=6.58, \eta_{\mathrm{p}}{ }^{2}=.08, \mathrm{p}=.012\right)$, demonstrating a greater RDR during the New_New versus Old_Old sequences.

\section{Discussion}

The current report yielded several new and important findings. First, the RDR is heavily influenced by decision incentives. Second, the RDR is also influenced by internally derived, local expectations. Finally, aside from the newly documented RDR incentive and expectancy effects, there was an additional, late-dilation response linked to feedback expectancy.

\section{Strength as a possible explanation}

One possibility is that the RDR may directly signal the overall strength of recognition evidence (e.g., Heaver \& Hutton, 2011; Otero et al., 2011; Papesh et al., 2012). However, the fact that subjects discriminate old and new probes perfectly well even when the RDR is eliminated through incentives, or because of prior decision outcomes, challenges this interpretation. As shown in the behavioral results, discrimination accuracy was not altered by incentives or decision sequence effects (see Online Supplementary Material), even though the RDR ranged from prominent to essentially non-existent (Figs. 2 and 3). An analogous decoupling of the RDR and discrimination performance was documented by Mill, O'Connor, and Dobbins (2016). If, however, the dilation response directly, and solely, reflected recognition strength, then discrimination should have been poor under situations in which the RDR was eliminated.

\section{Effort as a possible explanation}

A second possibility is that the RDR might reflect greater cognitive effort during hits than correct rejections (e.g., 




Fig. 3 Illustration of the effects of the two immediately preceding decisions on the current recognition dilation response (RDR). Sequences are coded with the first label representing the decision two trials back, and the second label representing the decision one trial back. Dilation during hits (HIT) is shown in blue and correct rejections (CR) in orange. Shading illustrates \pm 1 SEM
Papesh et al., 2012; Võ et al., 2008). For example, because hits (unlike correct rejections) can be accompanied by recollection of prior context, perhaps they elicit "additional retrieval or post-retrieval processes" or because "recollection is conceived of as a slower, more demanding process" hits may require greater effort (Võ et al., 2008, p. 138).

However, this interpretation is strained because not only are hits generally more certain than correct rejections, they are also generally rendered more quickly. Thus, positing they are more effortful means the above hypothetical evaluative or monitoring processes carry no behavioral costs. Moreover, Otero et al. (2011) demonstrated that greater depth of processing increases the RDR, and it is well established that deeper processing results in greater accuracy (viz., hit rates), certainty, and speed of recognition. Hence, holding that hits require greater cognitive effort than correct rejections despite these findings would completely detach the construct of "effort" from measurable choice behavior, defining effort solely in terms of pupil dilation. This is a reverse inference that would only be valid only if there was a single cause of pupil dilation during cognition.

However, aside from Kahneman's (1973) warning not to conflate dilations linked to deliberative cognition and orienting responses, the current data illustrate that fully dissociable dilation phenomena can arise within a single study (Fig. 2). More specifically, the RDR occurs early in the trials, reflects a greater dilation for hits than correct rejections, and is moderated by incentives regardless of the presence (Experiment 1) or absence (Experiment 2) of performance feedback. It is also moderated by local expectations (Fig. 3). In contrast, the feedback expectancy dilation occurs late in the trials, for either hits or correct rejections, depending upon which is incentivized, and only occurs in Experiment 1, in which post-decision feedback was available. Given this, it would be inappropriate to label both as reflecting the same psychological construct (e.g., "effort"), thereby implying they have the same neurophysiological basis (for review, see Joshi \& Gold, 2020).
Nonetheless, the RDR and feedback expectancy dilation responses may both signal the recruitment of general attentional resources from a limited capacity pool and in this abstract sense, both would reflect general effort or load as outlined by Kahneman (1973). However, in order to predict when such responses occur and explain their dissociations, it is necessary to develop process models of each phenomenon at a lower of abstraction.

\section{A multi-process explanation}

The current report tested whether the RDR might reflect an orienting response, albeit one triggered by encountering mnemonic as opposed to perceptual information. Critically, an orienting account is not independent of memory strength since, when motivational significance and expectation are held constant, stronger signals are expected to yield larger orienting responses. Thus the orienting account is consistent with the finding that in the control condition the RDR varies across confidence levels (see Online Supplementary Material) (see also Papesh et al., 2012). However, under an orienting account knowing memory strength in isolation is insufficient to predict the magnitude of orienting since it is moderated by expectation and motivational significance (for review, see Nieuwenhuis et al., 2011) . Consistent with this, the current data show the RDR is robust when recognition conclusions (and hence positive recognition evidence) are incentivized and essentially absent when novelty conclusions are instead incentivized. In terms of expectations, the data also reveal that preceding runs of "New" conclusions amplify the RDR, whereas runs of "Old" conclusions minimize it ... behavior that is strongly anticipated from orienting response research. For example, in "oddball" paradigms, the P300 component of the orienting response is increased when several standards precede a current target, and decreased when several targets precede a current target (Squires et al., 1976).

What of cognitive effort? The fact that time-pressured cognitive processing is linked to dilation is not incompatible with the conclusion the RDR reflects an orienting response, 
because the orienting account explains the differential dilation for hits versus correct rejections. However, both hits and correct rejections generally elicit robust dilation in comparison to baseline (Figs. 2 and 3), which is consistent with the idea that both share common information processing demands (e.g., lexical identification, semantic access, response selection, etc.) that must be accomplished within the trial.

Finally, the current data also demonstrate a feedback expectancy response that is unlikely to reflect cognitive effort, since no deliberative cognition is required in this interval. Additionally, the feedback expectancy dilation is unlikely to reflect an orienting response since no information is introduced in this interval. Speculatively, this dilation response may be tied to learning processes that map decision criteria onto anticipated rewards (de Gee et al., 2014). This would be essential for learning feedback-based recognition decision biases, which are easily induced via subtle feedback alterations during recognition testing (Han \& Dobbins, 2008, 2009).

In summary, these data suggest that the RDR is an orienting response to mnemonic information. Its adaptive value may lie in the ability of orienting to disrupt ongoing processing such that behaviorally relevant stimuli can gain privileged access to consideration. Additionally, the data revealed a late-occurring feedback expectancy dilation that may be tied to feedback-learning. Overall, the data highlight the richness of the dilation response, but also the danger of reverse inferring a single, common cause to this physiological signal.

Supplementary Information The online version contains supplementary material available at https://doi.org/10.3758/s13423-020-01866-w.

Acknowledgements The author would like to thank Lisa Solinger for her experiment programming, and the collection and pre-processing of pupillometry data.

Contributions I. G. Dobbins developed the study concept and design, as well as performing the statistical analyses. Testing, data collection, and pre-processing were performed by L. A. Solinger. The manuscript was prepared by I. G. Dobbins.

\section{References}

Baddeley, A. D. (1997). Human memory: Theory and practice. Psychology Press.

Beatty, J. T. (1982). Task-evoked pupillary responses, processing load, and the structure of processing resources. Psychological Bulletin, 91(2), 276-292. https://doi.org/10.1037//0033-2909.91.2.276

Benjamini, Y., \& Hochberg, Y. (1995). Controlling the false discovery rate: A practical and powerful approach to multiple testing. Journal of the Royal Statistical Society: Series B (Methodological), 57(1), 289-300.

Bradley, M. M. (2009). Natural selective attention: Orienting and emotion. Psychophysiology, 46(1), 1-11. https://doi.org/10.1111/j.14698986.2008.00702.x
Dalmaijer, E. S. Mathôt, S. Van der Stigchel, S. (2014). PyGaze: An open-source, cross-platform toolbox for minimal-effort programming of eyetracking experiments. Behavior Research Methods 46 (4), 913-921.

de Gee, J. W., Knapen, T., \& Donner, T. H. (2014). Decision-related pupil dilation reflects upcoming choice and individual bias. Proceedings of the National Academy of Sciences, 111(5), E618E625.

Dobbins, I. G., \& Han, S. (2007). What constitutes a model of item-based memory decisions? Psychology of Learning and Motivation, 48, 95-144.

Failing, M. F., \& Theeuwes, J. (2014). Exogenous visual orienting by reward. Journal of Vision, 14(5), 6-6. https://doi.org/10.1167/14.5.6

Friedman, D. Hakerem, G. Sutton, S. Fleiss, J. L. (1973). Effect of stimulus uncertainty on the pupillary dilation response and the vertex evoked potential. Electroencephalography and Clinical Neurophysiology 34(5), 475-484.

Han, S., \& Dobbins, I. G. (2008). Examining recognition criterion rigidity during testing using a biased-feedback technique: Evidence for adaptive criterion learning. Memory \& Cognition, 36(4), 703-715. https://doi.org/10.3758/MC.36.4.703

Han, S., \& Dobbins, I. G. (2009). Regulating recognition decisions through incremental reinforcement learning. Psychonomic Bulletin \& Review, 16(3), 469-474. https://doi.org/10.3758/PBR.16.3.469

Heaver, B., \& Hutton, S. B. (2011). Keeping an eye on the truth? Pupil size changes associated with recognition memory. Memory, 19(4), 398-405.

Hess, E. H., \& Polt, J. M. (1964). Pupil Size in Relation to Mental Activity during Simple Problem-Solving. Science 143(3611): 1190-1192.

Joshi, S., \& Gold, J. I. (2020). Pupil Size as a Window on Neural Substrates of Cognition. Trends in Cognitive Sciences, 24(6), 466480. https://doi.org/10.1016/j.tics.2020.03.005

Kahneman, D. (1973). Attention and effort (Vol. 1063). Citeseer.

Kahneman, D., \& Beatty, J. (1966). Pupil diameter and load on memory. Science, 154(3756), 1583-1585.

Kucera, H., Kučera, H., \& Francis, W. N. (1967). Computational analysis of present-day American English. Brown university press.

Lawrence, M. A. (2016). ez: Easy Analysis and Visualization of Factorial Experiments. $R$ Package Version 4.4-0. https://CRAN.R-project. org $/$ package $=$ ez

Le Pelley, M. E., Mitchell, C. J., Beesley, T., George, D. N., \& Wills, A. J. (2016). Attention and associative learning in humans: An integrative review. Psychological Bulletin, 142(10), 1111.

Macmillan, N. A., \& Creelman, C. D. (2004). Detection theory: A user's guide. Taylor Francis.

Mandler, G. (1980). Recognizing: The judgment of previous occurrence. Psychological Review, 87(3), 252.

Mathôt, S., Fabius, J., Van Heusden, E., \& Van der Stigchel, S. (2018). Safe and sensible preprocessing and baseline correction of pupil-size data. Behavior Research Methods, 50(1), 94-106. https://doi.org/10. 3758/s13428-017-1007-2

Matsumoto, M., \& Hikosaka, O. (2009). Two types of dopamine neuron distinctly convey positive and negative motivational signals. Nature, 459(7248), 837-841. https://doi.org/10.1038/nature08028

Mill, R. D., O'Connor, A. R., \& Dobbins, I. G. (2016). Pupil dilation during recognition memory: Isolating unexpected recognition from judgment uncertainty. Cognition, 154, 81-94. https://doi.org/10. 1016/j.cognition.2016.05.018

Nieuwenhuis, S., Geus, E. J. D., \& Aston-Jones, G. (2011). The anatomical and functional relationship between the $\mathrm{P} 3$ and autonomic components of the orienting response. Psychophysiology, 48(2), 162175. https://doi.org/10.1111/j.1469-8986.2010.01057.x

Otero, S. C., Weekes, B. S., \& Hutton, S. B. (2011). Pupil size changes during recognition memory. Psychophysiology, 48(10), 1346-1353. 
Papesh, M. H., Goldinger, S. D., \& Hout, M. C. (2012). Memory strength and specificity revealed by pupillometry. International Journal of Psychophysiology, 83(1), 56-64. https://doi.org/10.1016/j.ijpsycho. 2011.10.002

Peirce, J. W. (2007). PsychoPy-Psychophysics software in Python. Journal of Neuroscience Methods 162 (1-2), 8-13.

R Core Team. (2013). R: A language and environment for statistical computing. R Foundation for Statistical Computing. https://www. R-project.org/

Robinson, D. (2017). broom: Convert statistical analysis objects into tidy data frames. $R$ Package Version 0.4, 2.

Sokolov, E. N. (1963). Higher Nervous Functions: The Orienting Reflex. Annual Review of Physiology, 25(1), 545-580. https://doi.org/10. 1146/annurev.ph.25.030163.002553

Sokolov, E. N., Spinks, J. A., Näätänen, R., \& Lyytinen, H. (2002). The orienting response in information processing. Lawrence Erlbaum Associates Publishers.

Squires, K. C., Wickens, C., Squires, N. K., \& Donchin, E. (1976). The effect of stimulus sequence on the waveform of the cortical eventrelated potential. Science, 193(4258), 1142-1146.
Võ, M. L.-H., Jacobs, A. M., Kuchinke, L., Hofmann, M., Conrad, M., Schacht, A., \& Hutzler, F. (2008). The coupling of emotion and cognition in the eye: Introducing the pupil old/new effect. Psychophysiology, 45(1), 130-140. https://doi.org/10.1111/j.14698986.2007.00606.x

Wickham, H. (2017). tidyverse: Easily Install and Load the'Tidyverse'. $R$ Package Version 1.2.1, 1(1). https://CRAN.R-project.org/package= tidyverse

Wickham, H. (2019). forcats: Tools for Working with Categorical Variables (Factors). $R$ Package Version 0.4.0. https://CRAN.Rproject.org $/$ package $=$ forcats

Open Practices Statement The main analysis scripts and data are available at the Open Science Framework, http://osf.io/pk3ut.

Publisher's note Springer Nature remains neutral with regard to jurisdictional claims in published maps and institutional affiliations. 